\title{
Computer aided technology applied in decoration design
}

\author{
Ling Su \\ Hankou University, China \\ 836457021@qq.com
}

Keywords: Decorate design; Computer aided decorative; Simulation technology.

\begin{abstract}
The paper constructed the object orientation system structure, summed up the methods to realize computer aided decorate design based on the technology of simulation technology and OpenGL technology. The testing result of this system showed that the method of system building is feasible, and could be gone deep to study. This paper Considered the requirements for decorate design in the field of decorate design in the computer aided industrial design system. It gave a general discuss on the theory, working principle and key technique in order to construct a computer aided decorative design system in the future.
\end{abstract}

\section{INTRODUCTION}

Simulation technology has close relationship with decoration when it has not entered into the era. As early as in the era of Da Vinci, the architects were willing to draw virtual space on the four walls of the construction, in the hope that people could feel more vast in the area of the construction. In 1930 's, people tried to use four frame movie projectors to have wall projection at different decorate corners within the interior space, which can make people feel and experience the changeable decorate space in this room. After 1980's, with the development of computer technology, people simulated decorate interior space and group building space in the CAVE system.

After entering the twenty-first century, simulation technology was usually used for the construction of the study group or restoration. Eindoven University of Holland once used simulation technology to design and consult, they developed a software package that was composed of a set of functions of CAD, called CAAD software, which can create, modify and provide a toolkit for visualization of decoration. It can support the input and output of Auto CADDXF file, moreover, it also can increase the dynamic behavior animation for objects in the virtual environment. Taking a domestic example, in 2003 one VR for the Forbidden City called The Forbidden City, Emperor's Palace was an immersive theater, which could accommodate 54 staff, the high resolution images projected were 50 feet wide, 14 feet high on the curved screen. Visitors could fly freely in the virtual Forbidden City by using the controller so that visitors could roam in the Forbidden City between Kangxi Dynasty and Qianlong Dynasty, who could have closest watch on the panorama of the Forbidden City.

\section{An Overview of Simulation Technology}

Simulation technology, computer graphics software technology as well as virtual simulation system build up 3D simulation model, which also has build up testing technology through a series of experiments on 3D-dimensional model. 3D dimensional simulation technology has the advantages of high efficiency, safety, which can be less affected by environmental conditions and constraints, according to the actual needs of the project, it has the advantages that can change time and ratio of model size. From the characteristics of computer's 3D-dimensional simulation technology, there are 3D features: the first feature is the interactivity using 3D dimensional softwares, followed by the feature of imagination, finally the feature of immersion. "Immersion refers to the degree that users are in the real virtual environment. The ideal virtual environment can make it difficult for users to distinguish the degree between true and false (such as 3D visual scenes which are changed because of the changeable view ), even beyond the reality, such as the realization may be more real than real lighting and sound effects and so on." 
"Interaction refers to the degree of an object model that can be operated bu users within 3D virtual environment, as well as the degree of natural level that is got feedback from the environment (including real-time). If users can directly control the objects in the virtual environment, users also can move freely through the virtual environment when users are encountered with trees or walls in virtual scene, users will be covered by an object and they have to stop the motion."

Imagination refers to users who are immersed in the multidimensional virtual space, they can rely on their own perception and cognitive abilities so as to increase spatial conception effect in a full range, who can play full role initiatively and form new concept.

\section{The Application of Technology in Decorate Design}

The Stage of Creating Decorate Design. We usually use "two-dimensional" graphics to express 3D-dimensional objects, however, it is a simplification for two-dimensional information, and there is inevitably simplification and abstraction. The viewer can not have normal perspective to experience the decorate space, who can not acquire the real person's true feelings in the future. However, by using virtual reality, it can make the space of buildings be fully reflected in the decorate creation stage, so the decorate design of the space experience is more interactive and flexible, at the same time, the spirit of the place can be shown and expressed as much as possible, thus, the authenticity is greatly strengthened. In the virtual reality system, the actual feeling of be personally on the scene can show the proportion of the layout in decoration, which can be refined by adjusting the schemes of the designing elements. Immersed in the future construction, the designer thinking can be no longer interrupted and they can not wait so long time, creativity and inspiration will be greatly stimulated, therefore, the traditional methods can not compare with this method. As shown in fig 1.

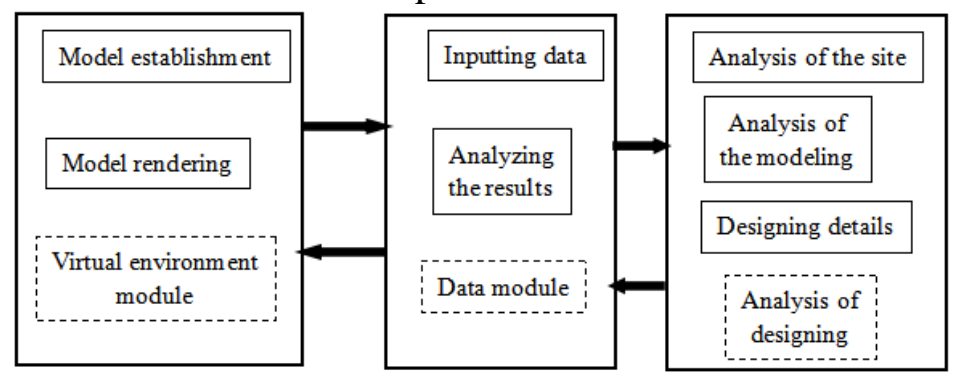

Fig. 1 Steps of aided decorate design for virtual reality

OpenGL technology in Decorate Design. Key Features. As an open, high-performance graphics and interactive visual treatment standards, OpenGL has the Features as follows:

Function rich: OpenGL library provides a rich graphics, image processing functions, including: all kinds of graphics rendering, lighting transformation, scene change, coordinate transformation, texture mapping, polygon division, and exchanging between the pixel storage format and double buffer etc.

Hardware independence: OpenGL is a graphics hardware-independent application interface. The user must begin to construct their own 3D-dimensional model from a point, line, surface and other basic graphic elements and perform 3D-dimensional real-time interactive operation.

Operation simple: OpenGL provides a lot of graphical functions which are Easy to understand. beginners can also use its powerful graphics capabilities to quickly build a 3D-dimensional graphics and interaction. It can run on the network, OpenGL is a client / server type. On the C/S decoration, the client sends OpenGL drawing commands, the server receives the command and executes a drawing by agreement.

Main functions Modeling: OpenGL graphics library provides the basic point, line, polygon drawing function, it also provides a complex 3D-dimensional objects, such as balls, cones, polyhedrons, teapots and complex curves and surfaces (such as Bezier, Nurbs curves, etc) drawing functions.

Transformation: The transformation function of OpenGL graphics library consists of two parts: basic transformation and projection transformation. Basic transformations Includes translation, rotation, ratio and mirror, projection transformation Includes parallel projection (orthographic projection) and perspective projection transformation. 
Color mode setting: OpenGL color mode Includes RGBA mode and color index.

Light and material settings: OpenGL light has Emitted Light, Ambient Light, Diffuse Light and Specular Light. The material is represented as the light reflectance.

Texture Mapping: The realistic surface details of an object can be expressed by the function of OpenGL texture mapping.

Bitmap display and image enhancement: OpenGL provides the basic image features pixel Copying and Reading, in addition, it also provides special image effects processing including blending, antialiasing and fog, which can enhance the graphics display effect

Double Buffering: OpenGL uses the front buffer and back buffer alternate scene technology, in short, back buffer computing scenarios, generate screen display, front buffer display picture which has been painted in the back buffer.

Special effects: OpenGL can achieve special effects such as Depth Cue, Motion Blur and other special effects.

The Performance Stage of Decorate Design. Simulation technique can provide designers more opportunities to experience man-machine program with multi-angle, so as to experience free multidimension interaction. People can choose to observe the building with static state, or in a variety of forms of motions to experience decorate space. At the same time, it also can have real-time comparison between different schemes, then after comparing, making judgment and choice. Moreover, the system can also simulate the sun light, as well as the related equipments and other facilities, which can make the overall expression of the building more comprehensive, real, scientific and convincing.

Interaction with other Designing Links. Using numerical simulation technology, it can be used to study and review the design of other links. Such as the simulation of wind environment of high-story building, so as to design the lower part and adjust the building's outer space, thus, the wind environment of high-story building's surrounding can be improved. In addition, it also can verify the safety of fire compartment, the control of smoke, evacuation routes and so on. In short, today, simulation technology can give support to the decorate design from the aspects of the professional integration, which can consider comprehensively with different majors, so as to make the building to be designed more perfectly.

\section{CONCLUSION}

In terms of decoration means, should be combined with surface rendering, volume rendering and vector drawing, and other forms of drawing way to richer, intuitive decoration environment information in the sphere.

\section{Reference}

[1] cheng Lin, product molding materials and technology, Beijing institute of technology press, 1991.6

[2] Renke He, history of industrial design, Beijing institute of technology press, 1996.8.

[3] Fangjie Tao, Fuyan Liu, Gang Liu, Large-Scale Interacting Particle System Simulation Using OpenGL [J], Proceedings of the International Conference on Information Engineering and Applications, 2013, Lecture Notes in Electrical Engineering Volume 218: 789-795.

[4] Miao Song, Peter Grogono, An LOD Control Interface for an OpenGL-based Softbody Simulation Framework[J], Innovations and Advances in Computer Sciences and Engineering, 2010, 539543.

[5] Yang Chao, Research on electromagnetic environment modeling and rendering three-dimensional method of virtual battlefield [D], Changsha: National University of Defense Technology, 2010.

[6] Liu Fang, Huang Mengcheng, Liu Xuehui, etc., based on efficient rendering of transparent barrel dynamic fusion phenomenon [J], Computer Aided Design and Computer Graphics, 2010,22 (3): 382-389. 